\title{
Nonlinear Predictive Sliding Mode Control for Active Suspension System
}

\author{
Dazhuang Wang $\mathbb{D}^{1},{ }^{1}$ Dingxuan Zhao $\mathbb{D},{ }^{1,2}$ Mingde Gong, ${ }^{2}$ and Bin Yang ${ }^{1}$ \\ ${ }^{1}$ School of Mechanical Science and Engineering, Jilin University, Changchun 130025, China \\ ${ }^{2}$ Collage of Mechanical Engineering, Yanshan University, Qinhuangdao 066004, China \\ Correspondence should be addressed to Dingxuan Zhao; zdx@ysu.edu.cn
}

Received 6 September 2017; Revised 15 February 2018; Accepted 20 March 2018; Published 2 May 2018

Academic Editor: Carlo Trigona

Copyright (c) 2018 Dazhuang Wang et al. This is an open access article distributed under the Creative Commons Attribution License, which permits unrestricted use, distribution, and reproduction in any medium, provided the original work is properly cited.

\begin{abstract}
An active suspension system is important in meeting the requirements of the ride comfort and handling stability for vehicles. In this work, a nonlinear model of active suspension system and a corresponding nonlinear robust predictive sliding mode control are established for the control problem of active suspension. Firstly, a seven-degree-of-freedom active suspension model is established considering the nonlinear effects of springs and dampers; and secondly, the dynamic model is expanded in the time domain, and the corresponding predictive sliding mode control is established. The uncertainties in the controller are approximated by the fuzzy logic system, and the adaptive controller reduces the approximation error to increase the robustness of the control system. Finally, the simulation results show that the ride comfort and handling stability performance of the active suspension system is better than that of the passive suspension system and the Skyhook active suspension. Thus, the system can obviously improve the shock absorption performance of vehicles.
\end{abstract}

\section{Introduction}

When a vehicle is running, the unevenness of the road seriously affects the comfort and handling stability of the vehicle ride, which influences the speed of the vehicle and even damages the vehicle parts. The suspension system of the vehicle can buffer the vibration caused by uneven road surface and transmit the braking and driving forces between the wheel and road surface. When the vehicle driving conditions change, the suspension system can withstand the forces and moments generated in different directions. Generally, the vehicle mainly uses the passive suspension system to reduce vibration; however, the spring stiffness and damping coefficient of the passive suspension are fixed, which cannot adapt to complex and varied road conditions and meet the ride comfort and handling stability requirements. In this case, the active suspension system emerges.

The active suspension system includes an actuator and a matching sensor and control unit that can input force and displacement to the suspension system based on the passive suspension system. In the control process, the active suspension system adjusts the output of the active suspension actuator according to the real-time changes of the road input and the vehicle status, thereby canceling the influence of the road and obtaining a good shock absorption effect while controlling the height and attitude.

A number of in-depth studies have proposed control methods for active suspension control to meet the ride comfort and handling stability requirements [1-8]. For example, [4] considered the influence of different vehicle speeds and road conditions and designed two separate fuzzy controllers for front and rear suspensions. Reference [5] developed a saturated adaptive robust control system for active suspension in response to uncertainties and the possible actuator saturation in systems; [6] provided an enhanced active suspension, established a fuzzy model, and used the fuzzy adaptive sliding mode control to verify the effectiveness of the model. Reference [7] established a dual objective controller for nonlinear active suspension systems with large classes of road profiles through simulation and experimentation. Reference [8] considered the complex nonlinearity of the active suspension system with the hydraulic actuator and designed 
an output feedback control with a high gain observer to estimate the nonlinearity. Although some types of control method are applied in the active suspension control, some traditional control methods do not consider the nonlinear effects and uncertainties of the active suspension system and cannot overcome the control process of time variability and uncertainty. This condition results in the application of such control method limitations.

Model predictive control (MPC) is an optimal control algorithm with distinct characteristics based on a model, rolling optimization, and feedback correction. The first MPCs were proposed in [9] in the 1970s to address the increasing control requirements that traditional single-loop controllers cannot meet. MPC has also been widely involved in the control of nonlinear systems and has achieved good results [10-12]. For example, [13-15] presented a good solution to nonlinear systems involved in the constrained nonlinear systems through the design of the nonlinear model predictive control (NMPC). Reference [16] designed a robust predictive controller based on the Lyapunov function and solved the problem of constraint and uncertain parameters in the nonlinear system, which guarantees robust performance; [17] developed a NMPC based on Carleman approximation for the nonlinear dynamic constraints. MPC has low requirements on modeling and successfully overcomes the shortcomings of the modern control theory model. MPC adopts the idea of optimal control and uses scrolling finiteperiod optimization instead of invariant global optimization. The active suspension system with uncertain factors and input-output constraints can be solved through MPC.

This paper consists of five parts. Section 1 is the introduction, which describes the active suspension and control systems and indicates the limitations of the controllers in solving the previously mentioned problems. Section 2 discusses the dynamic modeling of the nonlinear full-vehicle active suspension system. Section 3 presents the design of the predictive controller. The approximate expansion model of the active suspension system is developed in the rolling prediction time domain, and the predictive sliding mode controller of the nonlinear active suspension system is deduced by defining the performance evaluation function. For the complex nonlinear terms in the expansion, the fuzzy adaptive control is designed to increase the robustness of the control system. Section 4 presents the simulation analysis of the control system. Finally, Section 5 concludes the paper. The analysis results show that the active suspension can meet the vehicle requirements through the MPC, which can be compared with the passive suspension in improving system performance.

\section{Nonlinear Model for Active Suspension System}

2.1. The Full-Car Active Suspension System Model. The sevendegree-of-freedom automotive active suspension system model is shown in Figure 1. This model consists of four vertical unsprung masses and the degrees of freedom are due to pitch, roll, and vertical motions of the mass center $[18,19]$.
The variables of the active suspension model are shown in Variables of Active Suspension Model.

According to Newton's second law, the vertical motion of the body centroid, body pitching, and roll rotation equations can be obtained as follows $[19,20]$ :

$$
\begin{aligned}
& m \ddot{Z}=-F_{1}-F_{2}-F_{3}-F_{4}, \\
& J_{y} \ddot{\theta}=a\left(F_{1}+F_{2}\right)-b\left(F_{3}+F_{4}\right), \\
& J_{x} \ddot{\varphi}=\frac{l}{2}\left(-F_{1}+F_{2}-F_{3}+F_{4}\right),
\end{aligned}
$$

where $F_{i}(i=1,2,3,4)$ acts as the forces between the suspensions and the vertical body. This force is expressed as

$$
F_{i}=F_{k i}+F_{c i}-U_{i}
$$

where $F_{k i}$ and $F_{c i}$ are the spring and the damper forces, respectively; the forces consider the nonlinear effects of the springs and dampers and can yield $[21,22]$

$$
\begin{aligned}
F_{k i}= & k_{s i}\left(z_{i}-z_{u i}\right)+k_{n i}\left(z_{i}-z_{u i}\right)^{3}, \\
F_{c i}= & c_{i 1}\left(\dot{z}_{i}-\dot{z}_{u i}\right)+c_{i 2}\left|\dot{z}_{i}-\dot{z}_{u i}\right| \\
& +c_{i 3} \sqrt{\left|\dot{z}_{i}-\dot{z}_{u i}\right|} \operatorname{sgn}\left(\dot{z}_{i}-\dot{z}_{u i}\right) .
\end{aligned}
$$

Newton's law states that the dynamic differential equations of mass and spring mass can be listed separately.

$$
m_{u} \ddot{z}_{u}-F_{k}-F_{c}+F_{t}=-U,
$$

where $F_{t}$ is the force generated for the tire using the traditional point contact tire model, as follows:

$$
F_{t i}=k_{t i}\left(z_{u i}-z_{r i}\right)
$$

According to the spatial motion law of the rigid body, the dynamic relationship among the four suspension systems, including the body connection points, body centroid vertical movement, pitching rotation, and roll rotation, can be expressed as

$$
\begin{aligned}
& z_{1}=z-a \sin \theta+\frac{l}{2} \sin \varphi, \\
& z_{2}=z-a \sin \theta-\frac{l}{2} \sin \varphi, \\
& z_{3}=z+b \sin \theta+\frac{l}{2} \sin \varphi, \\
& z_{4}=z+b \sin \theta-\frac{l}{2} \sin \varphi .
\end{aligned}
$$

As a rigid body structure, the pitch and roll angles of the vertical body assume change in a small angle range. The following equations are set:

$$
\begin{aligned}
& \theta \approx \sin \theta, \\
& \varphi \approx \sin \varphi .
\end{aligned}
$$




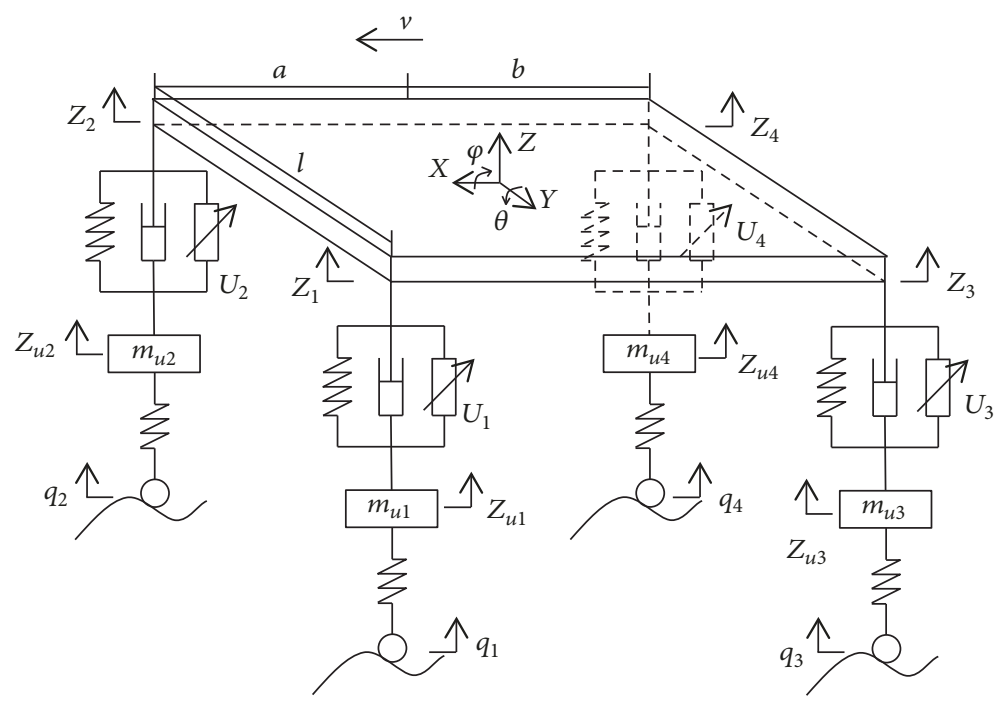

FIGURE 1: 7-DOF model of active suspension system.

The following equations are obtained when models (3) to (5) are applied in the dynamic differential equations ((1) to (2)). The state equation is established, and the system state vector $X$ is defined as

$X$

$$
=\left[\begin{array}{llllllllllllll}
z & \theta & \varphi & z_{u 1} & z_{u 2} & z_{u 3} & z_{u 4} & \dot{z} & \dot{\theta} & \dot{\varphi} & \dot{z}_{u 1} & \dot{z}_{u 2} & \dot{z}_{u 3} & \dot{z}_{u 4}
\end{array}\right]^{T} .
$$

The active suspension system can be expressed as

$$
\dot{X}=A X+B U+E Q+f_{n},
$$

where $Q$ is the road input vector of four tires, $A, B$, and $E$ divided into coefficient matrices and $f_{n}$ is the nonlinear term matrix.

System output $Y$ is defined as

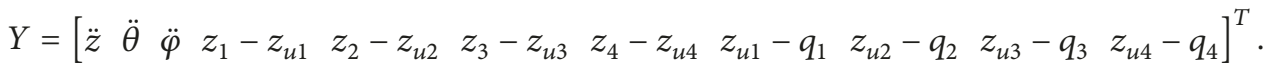

The output equation of the system is

$$
Y=C X+D Q+g_{n}
$$

where $C$ and $D$ are coefficient matrices, $g_{n}$ is the nonlinear term matrix, and matrices $A, B, C, D$, and $E$ can be derived from (1)-(5).

2.2. The Random Road Input Model. In the studies of active suspension control, the vibration generated inside a vehicle body is usually ignored, and the unevenness of the road surface is the most important factor that affects the vehicle ride comfort. The model of random road surface is generally selected as the most commonly used in simulation, so that more results and significance can be obtained. To study the nonlinear active suspension control system, a road input time domain model is established [23].

The vibration of the vehicle body is not only related to the road roughness but also related to the speed of the vehicle. When the vehicle travels at a speed $v$, we can get the following equation:

$$
f=v \cdot n,
$$

where $f$ is the time frequency which represents the number of waves contained in a time unit.

The power spectral density of road roughness can be expressed as

$$
\begin{aligned}
& D_{q}(f)=\frac{1}{v} D_{q}(n), \\
& D_{q}(n)=D_{q}\left(n_{0}\right)\left(\frac{n}{n_{0}}\right)^{-w},
\end{aligned}
$$

where $D_{q}\left(n_{0}\right)$ denotes the road roughness coefficient.

When $w=2$,

$$
D_{q}(f)=D_{q}\left(n_{0}\right) n_{0}^{2} \frac{v}{f^{2}},
$$

where $n_{0}$ is the standard spatial frequency.

Set $W(t)$ as the white noise signal. Then, we can obtain the transfer function

$$
H(j w)=\frac{2 \pi n_{0} \sqrt{D_{q}\left(n_{0}\right) v}}{j w+w_{0}} .
$$


The pavement contour function is obtained by passing the low-pass filter with function $H$. We can obtain the following stochastic pavement model:

$$
\dot{q}(t)=-2 \pi f_{0} v q(t)+2 \pi n_{0} \sqrt{D_{q}\left(n_{0}\right) v} w(t),
$$

In (17), $f_{0}$ is the road spatial cutoff frequency.

\section{MPC}

3.1. Approximate Expansion for MPC. Before the controller design, we assume that the system is dynamically stable and the signals are continuously different for time $t[24,25]$. In order to simplify the calculation,

$$
\begin{aligned}
& \frac{\partial C X}{\partial x} \dot{x}=L_{C}, \\
& \frac{\partial B U}{\partial u} \dot{u}=N_{B} .
\end{aligned}
$$

The $\rho$-step derivative of the system output $Y$ is

$$
\begin{aligned}
& \dot{y}=\frac{\partial C X}{\partial x} \dot{x}=L_{C}, \\
& \ddot{y}=\frac{\partial}{\partial x}\left(\frac{\partial C X}{\partial x} \dot{x}\right) \dot{x}=L_{C}^{2}, \\
& \vdots \\
& y^{(\rho)}=L_{C}^{(\rho)}, \\
& y^{(\rho+1)}=\frac{\partial L_{C}^{(\rho)}}{\partial x} \dot{x}+\frac{\partial L_{C}^{(\rho)}}{\partial u} \dot{u}=L_{C}^{(\rho+1)}+N_{B} L_{C}^{(\rho)}, \\
& y^{(\rho+2)}=\frac{\partial L_{C}^{(\rho+1)}}{\partial x} \dot{x}+\frac{\partial L_{C}^{(\rho+1)}}{\partial u} \dot{u}+\frac{\partial N_{B} L_{C}^{(\rho)}}{\partial x} \dot{x} \dot{u} \\
& \quad+\frac{\partial N_{B} L_{C}^{(\rho)}}{\partial u} \dot{u}^{2}+N_{B} L_{C}^{(\rho)} \ddot{u}=L_{C}^{(\rho+2)}+N_{B} L_{C}^{(\rho)} \ddot{u} \\
& \quad+Q_{1}(x, u, \dot{u}),
\end{aligned}
$$

where

$$
\begin{aligned}
Q_{1}(x, u, \dot{u})= & \frac{\partial L_{C}^{(\rho+1)}}{\partial u} \dot{u}+\frac{\partial N_{B} L_{C}^{(\rho)}}{\partial x} \dot{x} \dot{u}+\frac{\partial N_{B} L_{C}^{(\rho)}}{\partial u} \dot{u}^{2} \\
& +\frac{\partial N_{B}^{(\rho+1)}}{\partial u} \dot{u} .
\end{aligned}
$$

Repeating these steps yields

$$
\begin{aligned}
y^{(n)}= & L_{C}^{(n)}+N_{B} L_{C}^{(\rho)} u^{(n-\rho)} \\
& +Q_{n-\rho-1}\left(x, u, \dot{u}, \ldots, u^{(n-\rho-1)}\right) .
\end{aligned}
$$

To aim at nonlinear active suspension system (10)-(12), the following sliding surface is defined as

$$
\sigma_{i}=e_{i}^{(\rho-1)}+k_{i, \rho-1} e_{i}^{(\rho-2)}+\cdots+k_{i, 0} \int_{0}^{t} e_{i} d \tau,
$$

where $e_{i}=y_{i}-y_{r i}(i=1, \ldots, m)$ is the tracking error for the system, $y_{r i}$ is a smooth reference trajectory, and $k_{i, j}(j=$ $1, \ldots, \rho-1)$ is a sliding surface design parameter.

To converge $e_{i}$ to 0 when the slip surface $\sigma_{i}=0, k_{i, j}$ can be guaranteed to have the characteristic root corresponding to the following polynomial:

$$
p_{i}(\lambda)=\lambda_{i}^{(\rho)}+k_{i, \rho-1} \lambda_{i}^{(\rho-1)}+\cdots+k_{i, 0} \lambda_{i} .
$$

With $\sigma=\left[\sigma_{1}, \sigma_{2}, \ldots, \sigma_{m}\right]$, the sliding surface $\sigma$ is guided along the trajectory of the system:

$$
\begin{aligned}
\dot{\sigma}= & L_{C}^{(n)}+N_{B} L_{C}^{(\rho)} u^{(n-\rho)} \\
& +Q_{n-\rho-1}\left(x, u, \dot{u}, \ldots, u^{(n-\rho-1)}\right)-Y_{r}^{n}+Z_{k},
\end{aligned}
$$

where

$$
\begin{aligned}
Y_{r}^{(n)} & =\left[\begin{array}{c}
y_{r 1}^{(n)} \\
y_{r 2}^{(n)} \\
\vdots \\
y_{r m}^{(n)}
\end{array}\right], \\
Z_{k} & =\left[\begin{array}{c}
z_{1} \\
z_{2} \\
\vdots \\
z_{k}
\end{array}\right], \\
z_{i} & =k_{i, \rho-1} e_{i}^{(\rho-1)}+\cdots+k_{i, 0} e_{i} .
\end{aligned}
$$

The predicted value of $\sigma(t+\tau)$ can be expressed as

$$
\begin{aligned}
& \sigma^{T}(t) \approx \sigma(t)+T \dot{\sigma}(t)=\sigma(t)+T\left[L_{C}^{(n)}\right. \\
& +N_{B} L_{C}^{(\rho)} u^{(n-\rho)}-Y_{r}^{n}+Z_{k} \\
& \left.+Q_{n-\rho-1}\left(x, u, \dot{u}, \ldots, u^{(n-\rho-1)}\right)\right] .
\end{aligned}
$$

Because of the existence of $Q_{n-\rho-1}$ nonlinear interference term, the response speed and control precision of the predictive sliding mode control are affected. $Q_{n-\rho-1}$ is approximated by considering the fuzzy approximation law, which relies on the law of adaptive control to compensate for the MPC.

3.2. Fuzzy Logic System (FLS). According to [26, 27], FLS using IF-THEN rules consists of the knowledge base, fuzzifier, fuzzy inference engine, fuzzy rules, and defuzzifier. The knowledge base for FLS comprises a collection of fuzzy IF-THEN rules of the following form:

$$
R_{i} \text { : If } x_{1} \text { is } A_{1}^{i} \text { and } \ldots \text { and } x_{n} \text { is } A_{n}^{i} \text {, then } y \text { is } B^{i} \text {, }
$$

where $x_{i}$ and $y$ are the input and output of the FLS. $A_{1}^{i} \cdots A^{i}{ }_{n}$ and $B^{i}$ are fuzzy sets, respectively, which are associated with the fuzzy membership functions. Define the FLS as

$$
Y(x)=\frac{\sum_{i=1}^{h} y^{i}\left(\prod_{j=1}^{n} F_{j}^{i}\left(x_{j}\right)\right)}{\sum_{i=1}^{h} \prod_{j=1}^{n} F_{j}^{i}\left(x_{j}\right)}=\omega^{T} \mu(x),
$$


where $F_{j}^{i}\left(x_{j}\right)$ is the membership function and $\omega^{T}$ is the basis function vector of FLS.

Set $\mu(x)$ as

$$
\mu(x)=\left[\mu_{1}(x), \mu_{2}(x), \ldots, \mu_{h}(x)\right],
$$

where

$$
\mu_{i}(x)=\frac{\prod_{j=1}^{n} F_{j}^{i}\left(x_{j}\right)}{\sum_{i=1}^{h}\left(\prod_{j=1}^{n} F_{j}^{i}\left(x_{j}\right)\right)} .
$$

In order to develop the leaning algorithms for FLS, the functional form of fuzzy membership function should be specified. The membership function used in this study is a Gaussian-shaped form $[28,29]$ :

$$
F_{j}^{i}\left(x_{j}\right)=\exp \left[-\frac{\left(x_{j}-p_{j}\right)^{2}}{2 q_{j}^{2}}\right] .
$$

According to the above formulas, which are used to approximate the compound interference term $Q_{n-\rho-1}$, the fuzzy system can be expressed as

$$
Q_{n-\rho-1}=\omega^{T} \mu(x) .
$$

For any given $x$ in compact set $\mu^{*}$, the optimal weight vector $\mu^{*}$ of the fuzzy system exists as [30]

$$
\mu^{*}=\arg \min _{\mu \in \Omega_{\theta}}\left[\sup \left\|Q_{n-\rho-1}-\omega^{T} \mu(x)\right\|\right] .
$$

The optimal weight vector $\mu^{*}$ is within the convex region:

$$
\Omega_{\theta}=\left\{\mu \mid\|\mu\| \leq M_{0}\right\},
$$

where $M_{0}>0$.

According to the above functions, the norm of the optimal weight $\theta^{*}$ is bounded

$$
\left\|\mu^{*}\right\| \leq \bar{\mu}
$$

where $\bar{\mu}>0$.

The fuzzy approximation error is defined as follows:

$$
\varepsilon=Q_{n-\rho-1}-\omega^{T} \mu^{*}(x) .
$$

To overcome the impact of the approximation error on the system and improve the performance of the entire system, a robust compensation term should be introduced.

Set the adaptive law as

$$
\dot{\theta}=\lambda_{\theta} \mu(x) \sigma .
$$

The error vector and the Lyapunov function are defined as follows:

$$
\begin{aligned}
V(\Phi) & =\Phi^{T} \bar{P} \Phi, \\
\Phi & =\left[\begin{array}{ll}
\sigma^{T} & \bar{\mu}^{T}
\end{array}\right]^{T} \in D_{\Phi},
\end{aligned}
$$

where $D$ is the domain of the augmented error and $\bar{P}$ is the weight matrix

$$
\bar{P}=\frac{1}{2}\left[\begin{array}{cc}
I_{\sigma} & 0 \\
0 & \lambda_{\mu}^{-1} I_{\mu}
\end{array}\right] .
$$

The Lyapunov function defined by (38) is derived from the system trajectory for time $t$

$$
\begin{aligned}
\dot{V}(\Pi)= & \sigma^{T} \dot{\sigma}+\frac{1}{\lambda_{\mu}} \tilde{\mu}^{T} \dot{\tilde{\mu}}, \\
\dot{V}(\Pi)= & \sigma^{T}\left(-T^{-1} \sigma+Q_{n-\rho-1}-\omega^{T} \mu(x)-\phi \operatorname{sign}(\sigma)\right) \\
& -\frac{1}{\lambda_{\mu}} \tilde{\mu}^{T} \dot{\tilde{\mu}} \\
\leq & -T^{-1} \sigma^{T} \sigma-\|\sigma\|(\phi-\omega) \\
& +\left(\sigma^{T} \varphi^{T}(x) \bar{\mu}-\frac{1}{\lambda_{\mu}} \bar{\mu}^{T} \dot{\mu}\right) .
\end{aligned}
$$

The adaptive law (32) can yield

$$
\sigma^{T} \dot{\sigma} \widetilde{\mu}-\frac{1}{\lambda_{\mu}} \widetilde{\mu}^{T} \dot{\tilde{\mu}}=0 .
$$

The synthesis of $=(41)-(42)$ can be obtained as follows:

$$
\dot{V} \leq-T^{-1} \sigma^{T} \sigma-\|\sigma\|(\phi-\omega) .
$$

3.3. Predictive Sliding Mode Control for Nonlinear Active Suspension. The nonlinear predictive control uses the control algorithm of the rolling optimization, and the optimal control quantity is determined by optimizing the performance index to achieve the purpose of the optimal tracking desired trajectory of the system output $Y$.

The performance index function of one predictive sliding mode control is considered as follows:

$$
J=\frac{1}{2}\left[\sigma^{T}(t+\tau) Q \sigma(t+\tau)+u^{T}(t+\tau) R u(t+\tau)\right],
$$

where $Q$ and $R$ are positive weight matrices and $\tau$ is a decimal number greater than zero.

Solving predictive control problems can be equivalent to solving

$$
\min J(x, u, t) .
$$

The performance index $J$ is derived from the control quantity $u$, which yields

$$
\frac{\partial J}{\partial u}=\frac{1}{2}\left[\left(\frac{\partial \sigma^{T}(t+\tau)}{\partial \sigma}\right)^{T} Q \sigma(t+\tau)+R u\right] .
$$

Given that

$$
\frac{\partial J}{\partial u}=0
$$


the predictive sliding mode control law is

$$
U_{P}=-L_{U} L_{C}^{\rho-1} T\left[\sigma+T\left(F(x)+Z_{k}-Y_{r}^{\rho}\right)\right]
$$

and the adaptive control law is

$$
U_{A}=-L_{U} L_{C}^{\rho-1}\left(Q_{n-\rho-1}+\varphi \operatorname{sign}(\sigma)\right) .
$$

Thus, the nonlinear robust adaptive control law based on MPC can be expressed as

$$
U=U_{P}+U_{A}
$$

By approximating the nonlinear active suspension system in the time domain, the controller can effectively reduce the model accuracy requirements and better adaptability to uncertain factors in the model. At the same time, due to the nonlinear functions in the approximation, the expansion is difficult to calculate. The uncertainties in the controller are approximated by the FLS to reduce the amount of calculation, and the adaptive controller increases the robustness of the control system.

\section{Simulation Results and Discussion}

Based on the simulation of the designed nonlinear predictive sliding mode control of active suspension and comparing it with the passive suspension and the Skyhook active suspension, Table 1 lists the parameters of the nonlinear active suspension system in the simulation.

The figures show that the predictive sliding mode control of the nonlinear active suspension has better effects in terms of passenger comfort and handling stability compared with the other suspension systems. The controller can obviously improve body acceleration, pitch acceleration, roll acceleration, and deflections of suspensions and tires during vehicle driving. Passenger ride comfort through body acceleration, pitch acceleration, and roll acceleration is shown in Figures 2-4. A comparison of active suspension with the passive suspension and traditional Skyhook controller shows that body acceleration fluctuations significantly reduced in Figure 2. At the same time, the simulation results show that the peak values of the vertical acceleration of the three suspension systems are $2.12 \mathrm{~m} / \mathrm{s}^{2}, 1.10 \mathrm{~m} / \mathrm{s}^{2}$, and $0.81 \mathrm{~m} / \mathrm{s}^{2}$, respectively, and the RMS values are $0.641 \mathrm{~m} / \mathrm{s}^{2}, 0.319 \mathrm{~m} / \mathrm{s}^{2}$, and $0.202 \mathrm{~m} / \mathrm{s}^{2}$, respectively. Meanwhile, as shown in Figures 3 and 4, the pitch and roll acceleration of the active suspension vehicle had a significant improvement compared with the passive suspension and Skyhook suspension system. In Figure 3, the peak values of the pitch acceleration are $7.45 \mathrm{rad} / \mathrm{s}^{2}, 3.94 \mathrm{rad} / \mathrm{s}^{2}$, and $2.10 \mathrm{rad} / \mathrm{s}^{2}$ and the $\mathrm{RMS}$ values are $2.170 \mathrm{rad} / \mathrm{s}^{2}, 1.247 \mathrm{rad} / \mathrm{s}^{2}$, and $0.661 \mathrm{rad} / \mathrm{s}^{2}$. In Figure 4, the peak values of the roll acceleration are $2.69 \mathrm{rad} / \mathrm{s}^{2}, 1.74 \mathrm{rad} / \mathrm{s}^{2}$, and $0.85 \mathrm{rad} / \mathrm{s}^{2}$, the RMS values are $0.951 \mathrm{rad} / \mathrm{s}^{2}, 0.576 \mathrm{rad} / \mathrm{s}^{2}$, and $0.322 \mathrm{rad} / \mathrm{s}^{2}$.

These results show that the designed active suspension system has a good effect on reducing the vibration of the vehicle and greatly improved the vehicle ride comfort during the driving process. Through the simulation of the suspension and tire deflections, the vehicle handling stability for
TABLE 1: Simulation parameters $(i=1,2,3,4)$.

\begin{tabular}{lc}
\hline Parameter & Value \\
\hline$m$ & $1600 \mathrm{Kg}$ \\
$m_{u}$ & $45 \mathrm{Kg}$ \\
$k_{s i}$ & $14000 \mathrm{~N} / \mathrm{m}$ \\
$k_{n i}$ & $1000 \mathrm{~N} / \mathrm{m}$ \\
$k_{t i}$ & $250000 \mathrm{~N} / \mathrm{m}$ \\
$c_{i 1}$ & $1600 \mathrm{Ns} / \mathrm{m}$ \\
$c_{i 2}$ & $350 \mathrm{Ns} / \mathrm{m}$ \\
$c_{i 3}$ & $350 \mathrm{Ns} / \mathrm{m}$ \\
$l$ & $1.5 \mathrm{~m}$ \\
$f_{0}$ & $0.01 \mathrm{~m}{ }^{-1}$ \\
$J x$ & $450 \mathrm{Kg} \cdot \mathrm{m}^{2}$ \\
$J y$ & $2100 \mathrm{Kg} \cdot \mathrm{m}^{2}$ \\
$a$ & $1.4 \mathrm{~m}$ \\
$b$ & $1.7 \mathrm{~m}$ \\
$\omega$ & 2 \\
$n$ & 5 \\
$\lambda_{\mu}$ & 0.6 \\
$j$ & 1,2 \\
$n_{0}$ & $0.1 \mathrm{~m}^{-1}$ \\
\hline
\end{tabular}

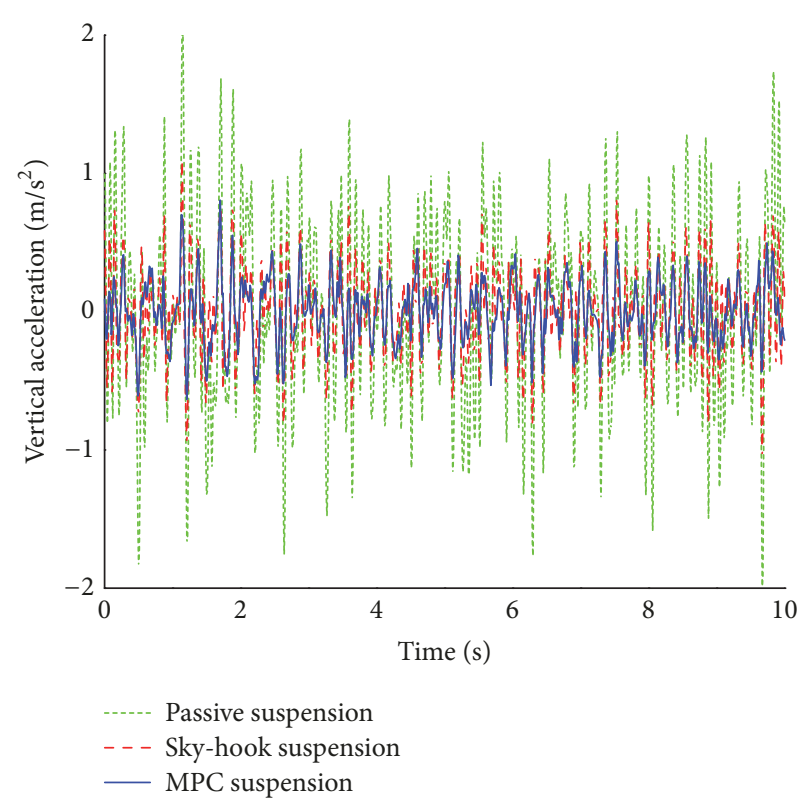

FIgURE 2: Vertical acceleration of car body.

active suspensions and passive suspension can be obviously displayed. The suspension deflections are shown in Figures 5 and 6, where the predictive sliding mode controller is shown to have a better performance than the others. Figures 7 and 8 show the improvement of the tire deflections. Moreover, the effect of the sliding mode predictive control of active suspension is shown to be significantly better than that of the other suspension systems (Figure 9). 


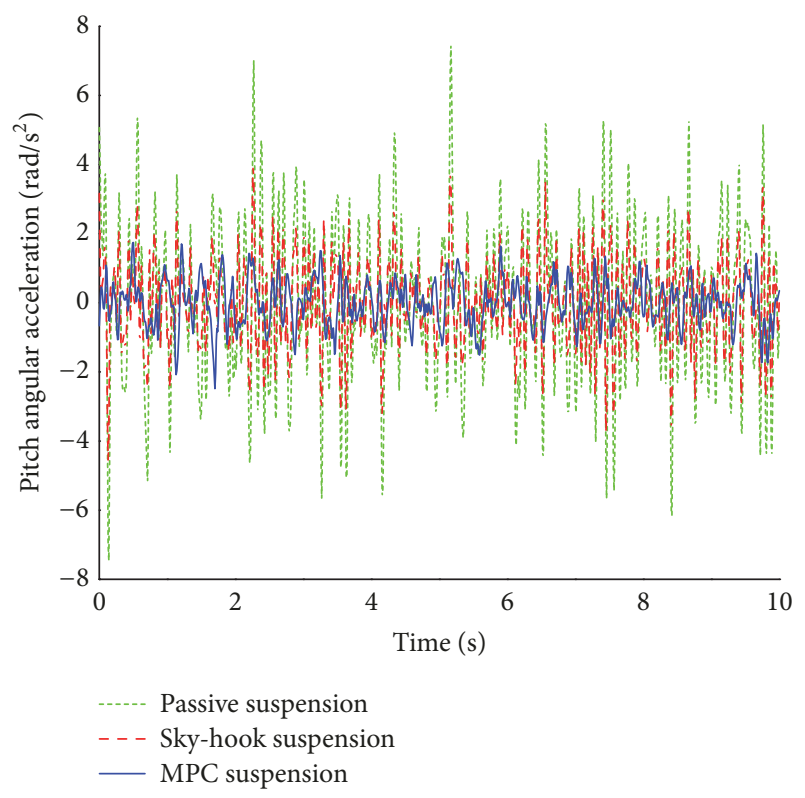

Figure 3: Pitch acceleration.

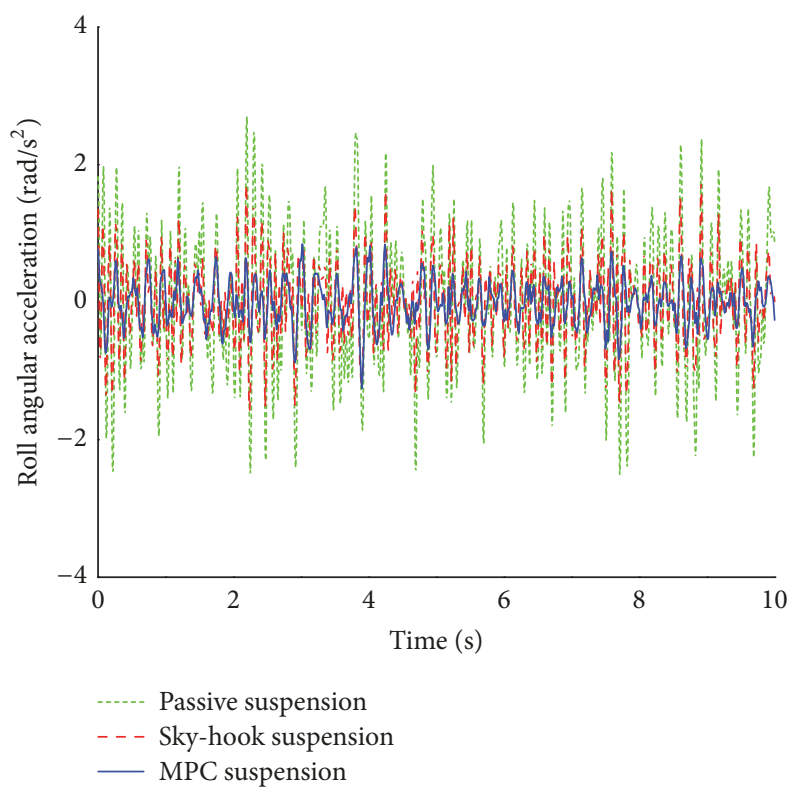

Figure 4: Roll acceleration.

In order to verify the control effects in the frequency domain, the energy spectral density figures of the vertical acceleration, pitch, and roll accelerations of the three suspension systems are established, as shown in Figures 10-12.

It can be seen from the figures that the amplitudes of the two active suspension systems have been significantly reduced. In the frequency range of $10-20 \mathrm{~Hz}$, the effect of MPC is better than that of Skyhook damping suspension, while in the low frequency range $0-5 \mathrm{~Hz}$, Skyhook damping active suspension system is better than MPC.

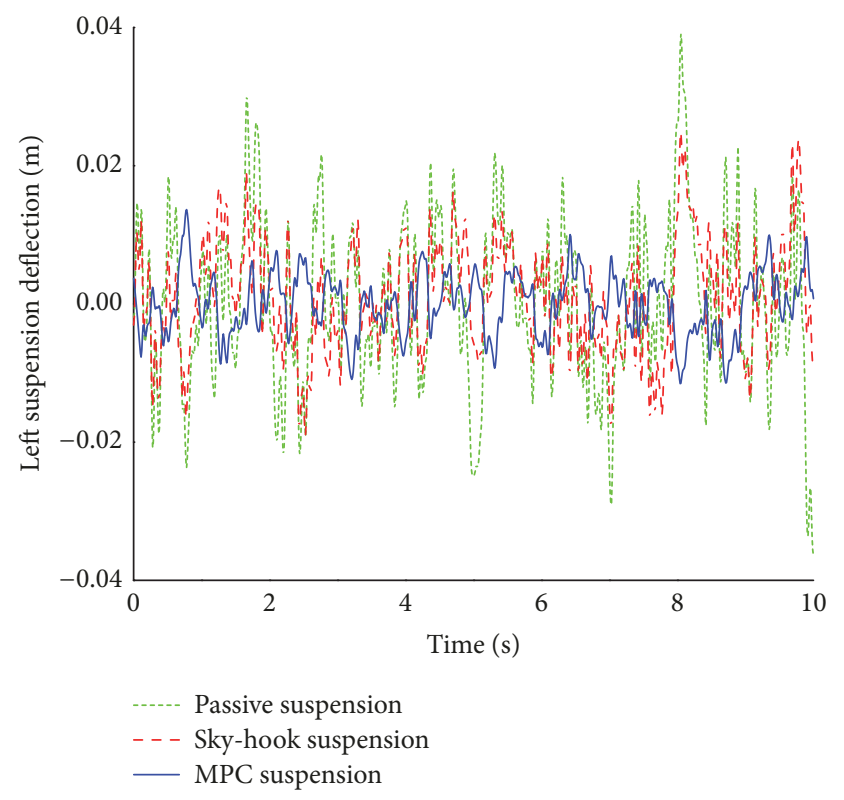

Figure 5: Deflection of the suspension (left).

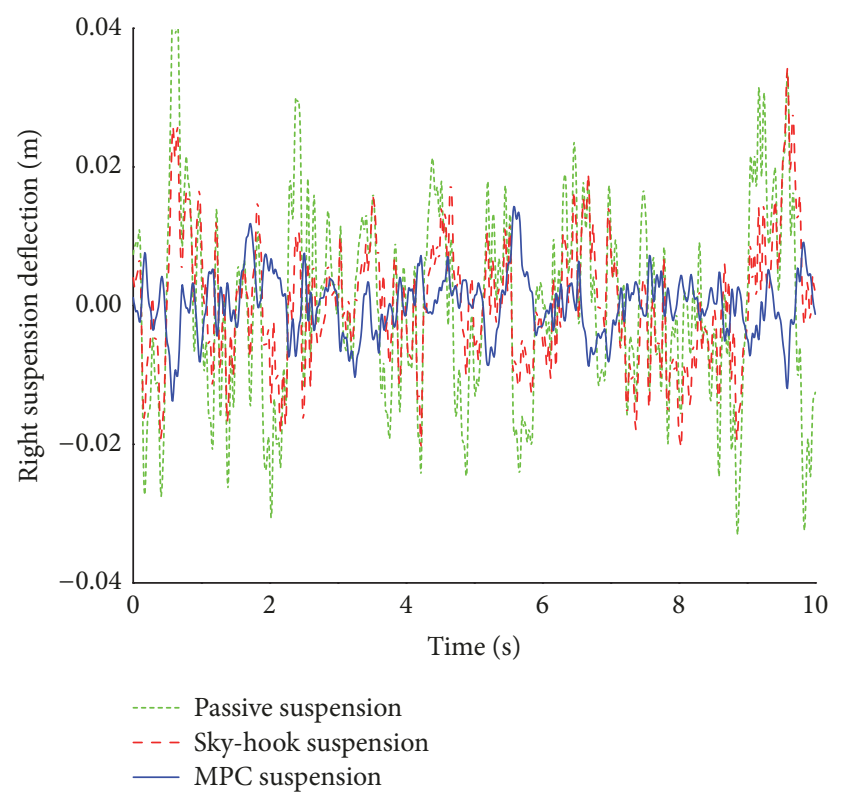

FIGURE 6: Deflection of the suspension (right).

\section{Conclusion}

This study establishes the nonlinear system dynamic model of active suspension, including the nonlinear effects of the spring and damper. Moreover, a nonlinear predictive sliding mode control is designed based on the approximate expansion in the time domain. FLS is applied to approximate the complex nonlinear term generated by the expansion. The approximation error is effectively suppressed by using the adaptive control to increase the robustness of the control system. The results show that the predictive sliding mode 


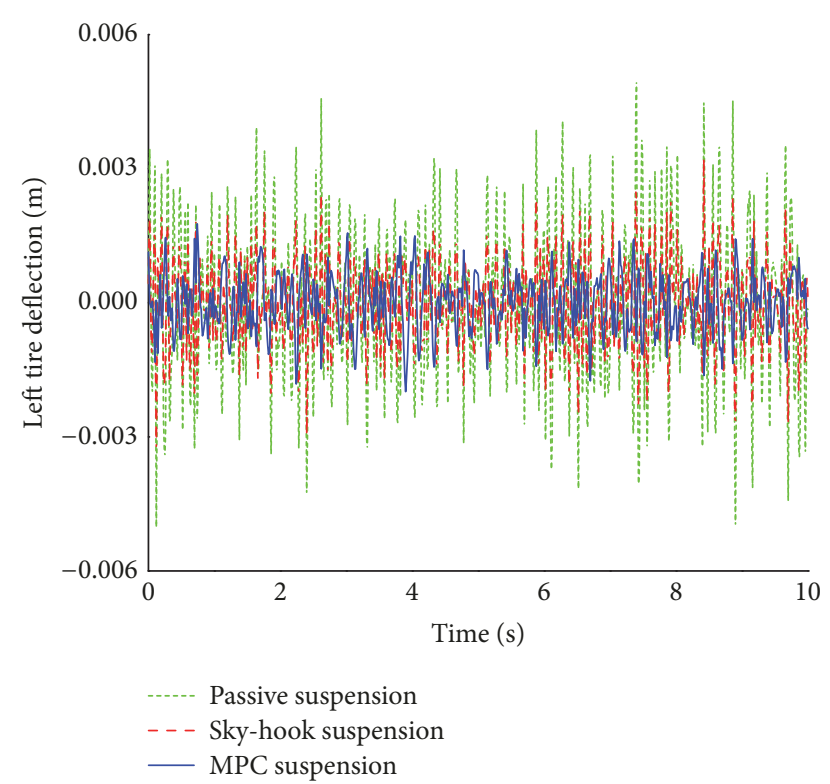

Figure 7: Deflection of the tire (left).

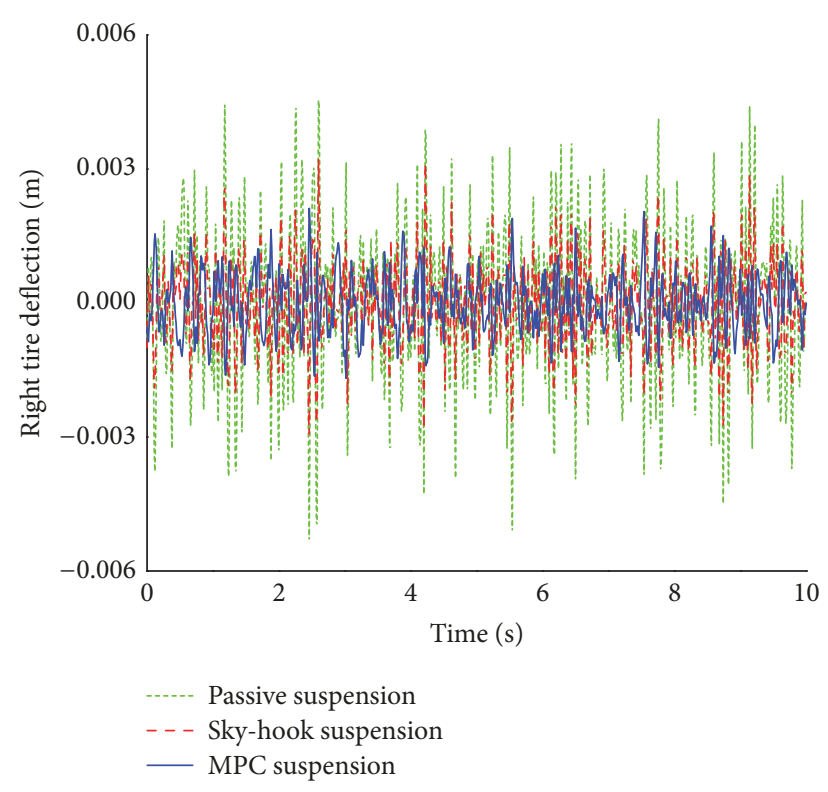

FIGURE 8: Deflection of the tire (right).

controller can effectively improve vehicle performance and reduce vehicle vibration compared with the passive suspension. The predictive sliding mode control based on the approximate expansion is less demanding for the accuracy of the original nonlinear active suspension model. Furthermore, the uncertainty of the model is greatly improved in the process of practical application.

\section{Variables of Active Suspension Model}

$m$ : Mass of the vehicle body

mui: Unsprung masses

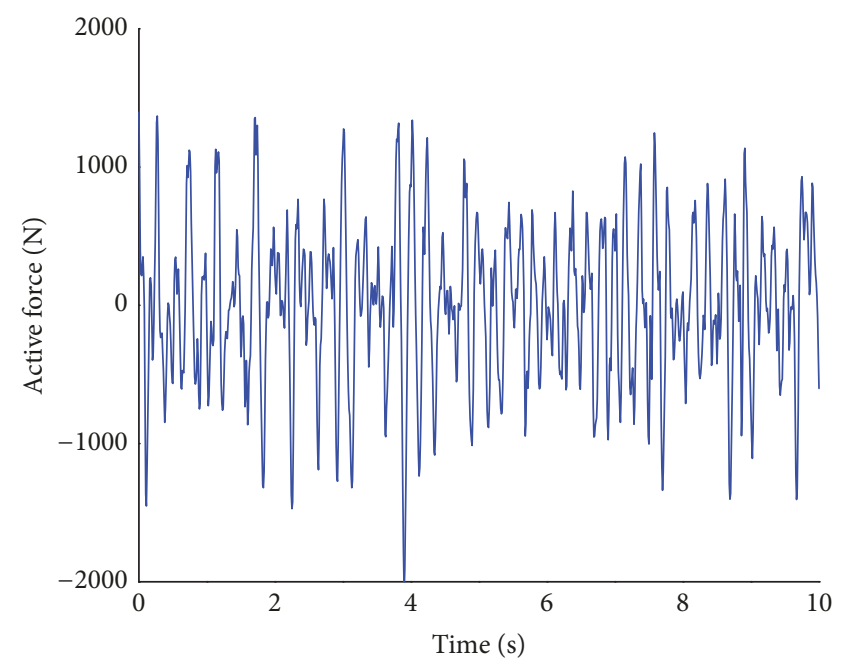

FIGURE 9: System state of active force.

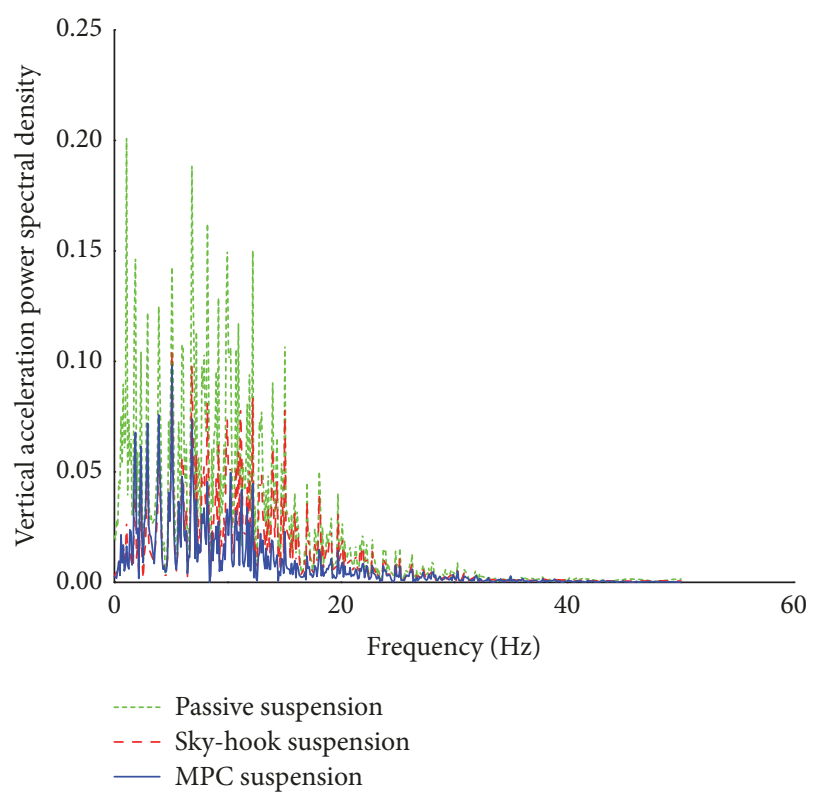

Figure 10: Vertical acceleration power spectral density.

$z:$ Displacement of the vehicle body

$z_{i}$ : Displacements between vehicle body and suspensions

$z_{u i}$ : Unsprung mass displacements

$q_{i}$ : Vertical road displacements

$a$ : Distance between center of mass and front suspension

$b$ : Distance between center of mass and rear suspension

l: Distance between left and right suspensions

$\theta$ : Pitch angle of the vehicle body

$\varphi$ : Roll angle of the vehicle body

$J x$ : Moment of inertia of the $X$-axis

$J y$ : Moment of inertia of the $Y$-axis 


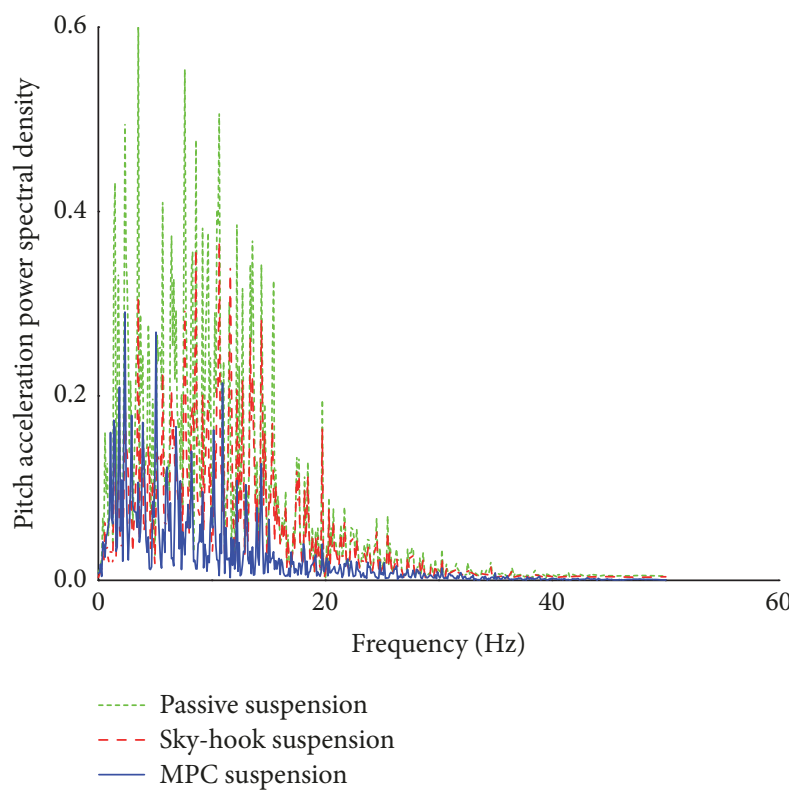

FIGURE 11: Pitch acceleration power spectral density.

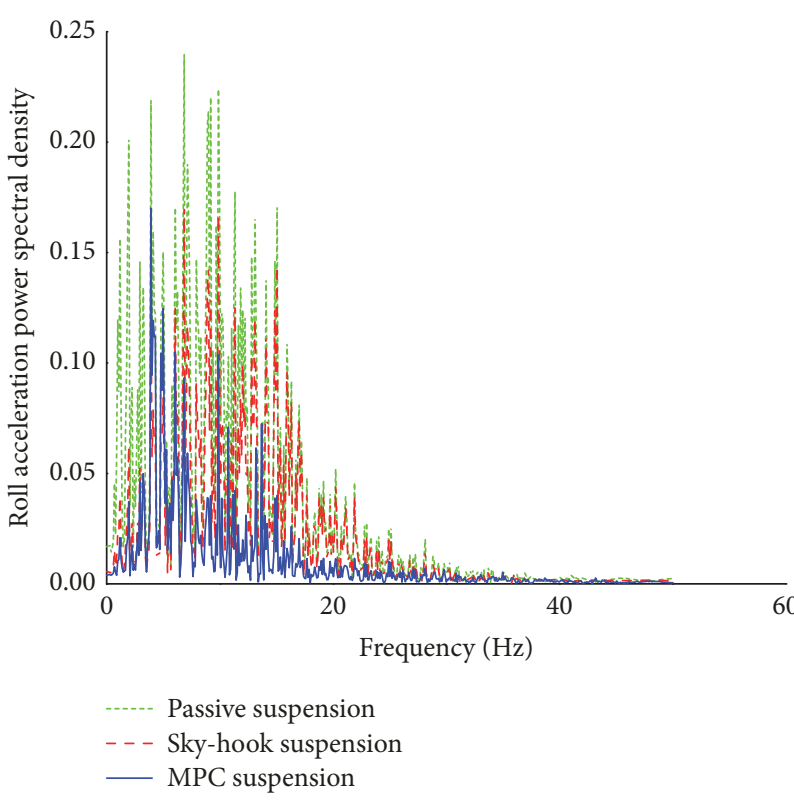

FIGURE 12: Roll acceleration power spectral density.

$k_{s i}$ : Stiffness coefficients of the springs

$k_{n i}$ : Nonlinear components of the spring

$k_{t i}$ : Stiffness coefficients of the tires

$c_{i 1}$ : Damping coefficients

$c_{i 2}$ : Damping modification coefficient

$c_{i 3}:$ Shock absorber nonlinear modification coefficient

$U i$ : Forces generated by actuators.

\section{Conflicts of Interest}

The authors declare that they have no conflicts of interest.

\section{Acknowledgments}

This work was supported by the National Key R\&D Program of China "Key Technology Research on Special Chassis and Suspension for High-mobility Emergency Rescue Vehicle (including Fire Fighting Vehicle)" (Project no. 2016YFC0802900).

\section{References}

[1] H. Li, H. Liu, H. Gao, and P. Shi, "Reliable fuzzy control for active suspension systems with actuator delay and fault," IEEE Transactions on Fuzzy Systems, vol. 20, no. 2, pp. 342-357, 2012.

[2] C. Gohrle, A. Schindler, A. Wagner, and O. Sawodny, "Design and vehicle implementation of preview active suspension controllers," IEEE Transactions on Control Systems Technology, vol. 22, no. 3, pp. 1135-1142, 2014.

[3] H. H. Pan, W. C. Sun, H. J. Gao, and J. Y. Yu, "Finite-time stabilization for vehicle active suspension systems with hard constraints," IEEE Transactions on Intelligent Transportation Systems, vol. 16, no. 5, pp. 2663-2672, 2015.

[4] M. Soleymani, M. Montazeri-Gh, and R. Amiryan, "Adaptive fuzzy controller for vehicle active suspension system based on traffic conditions," Scientia Iranica, vol. 19, no. 3, pp. 443-453, 2012.

[5] W. Sun, Z. Zhao, and H. Gao, "Saturated adaptive robust control for active suspension systems," IEEE Transactions on Industrial Electronics, vol. 60, no. 9, pp. 3889-3896, 2013.

[6] J. Lin, R.-J. Lian, C.-N. Huang, and W.-T. Sie, "Enhanced fuzzy sliding mode controller for active suspension systems," Mechatronics, vol. 19, no. 7, pp. 1178-1190, 2009.

[7] V. S. Deshpande, P. D. Shendge, and S. B. Phadke, "Nonlinear Control for Dual Objective Active Suspension Systems," IEEE Transactions on Intelligent Transportation Systems, vol. 18, no. 3, pp. 656-665, 2017.

[8] J. J. Rath, M. Defoort, H. R. Karimi, and K. C. Veluvolu, "Output Feedback Active Suspension Control with Higher Order Terminal Sliding Mode," IEEE Transactions on Industrial Electronics, vol. 64, no. 2, pp. 1392-1403, 2017.

[9] S. J. Qin and T. A. Badgwell, "An overview of industrial model predictive control technology," Control Engineering Practice, vol. 93, pp. 232-256, 1997.

[10] B. T. Stewart, S. J. Wright, and J. B. Rawlings, "Cooperative distributed model predictive control for nonlinear systems," Systems \& Control Letters, vol. 21, no. 5, pp. 698-704, 2011.

[11] J. Zhao, S. Zhou, and R. Zhou, "Distributed time-constrained guidance using nonlinear model predictive control," Nonlinear Dynamics, vol. 84, no. 3, pp. 1-18, 2016.

[12] F. Xu, H. Chen, X. Gong, and Q. Mei, "Fast nonlinear model predictive control on FPGA using particle swarm optimization," IEEE Transactions on Industrial Electronics, vol. 63, no. 1, pp. 310-321, 2016.

[13] T. Sun, Y. Pan, J. Zhang et al., "Robust model predictive control for constrained continuous-time nonlinear systems," International Journal of Control, pp. 1-16, 2017.

[14] X. Liu, Y. Shi, and D. Constantinescu, "Robust distributed model predictive control of constrained dynamically decoupled nonlinear systems: A contraction theory perspective," Systems \& Control Letters, vol. 105, pp. 84-91, 2017.

[15] B. Picasso, X. Zhang, and R. Scattolini, "Hierarchical model predictive control of independent systems with joint constraints," Automatica, vol. 74, pp. 99-106, 2016. 
[16] P. Mhaskar, N. H. El-Farra, and P. D. Christofides, "Robust hybrid predictive control of nonlinear systems," Automatica, vol. 41, no. 2, pp. 209-217, 2005.

[17] Y. Fang and A. Armaou, "Carleman approximation based quasianalytic model predictive control for nonlinear systems," AIChE Journal, vol. 62, no. 11, pp. 3915-3929, 2016.

[18] M. Yamashita, K. Fujimori, K. Hayakawa, and H. Kimura, "Application of Ho control to active suspension systems," Automatica, vol. 30, no. 11, pp. 1717-1729, 1994.

[19] H. J. Kim, H. S. Yang, and Y. P. Park, "Improving the vehicle performance with active suspension using road-sensing algorithm," Computers \& Structures, vol. 80, no. 18, pp. 1569-1577, 2002.

[20] W. Sun, H. Gao, and B. Yao, "Adaptive robust vibration control of full-car active suspensions with electrohydraulic actuators," IEEE Transactions on Control Systems Technology, vol. 21, no. 6, pp. 2417-2422, 2013.

[21] W. Sun, H. Pan, Y. Zhang, and H. Gao, "Multi-objective control for uncertain nonlinear active suspension systems," Mechatronics, vol. 24, no. 4, pp. 318-327, 2014.

[22] M. Dangor, O. A. Dahunsi, J. O. Pedro, and M. M. Ali, "Evolutionary algorithm-based PID controller tuning for nonlinear quarter-car electrohydraulic vehicle suspensions," Nonlinear Dynamics, vol. 78, no. 4, pp. 2795-2810, 2014.

[23] X. Dong, D. Zhao, B. Yang, and C. Han, "Fractional-order control of active suspension actuator based on parallel adaptive clonal selection algorithm," Journal of Mechanical Science and Technology, vol. 30, no. 6, pp. 2769-2781, 2016.

[24] W. H. Chen, "Predictive control of general nonlinear systems using approximation," IEE Proceedings Control Theory and Applications, vol. 151, no. 2, pp. 137-144, 2004.

[25] W.-H. Chen, D. J. Ballance, and P. J. Gawthrop, "Optimal control of nonlinear systems: a predictive control approach," Automatica, vol. 39, no. 4, pp. 633-641, 2003.

[26] L. Wang, "Stable adaptive fuzzy control of nonlinear systems," IEEE Transactions on Fuzzy Systems, vol. 1, no. 2, pp. 146-155, 1993.

[27] S.-C. Tong and H.-X. Li, "Fuzzy adaptive sliding-mode control for MIMO nonlinear systems," IEEE Transactions on Fuzzy Systems, vol. 11, no. 3, pp. 354-360, 2003.

[28] X. Zhao, P. Shi, and X. Zheng, "Fuzzy Adaptive Control Design and Discretization for a Class of Nonlinear Uncertain Systems," IEEE Transactions on Cybernetics, vol. 46, no. 6, pp. 1476-1483, 2016.

[29] Y. Li, S. Tong, and T. Li, "Hybrid Fuzzy Adaptive Output Feedback Control Design for Uncertain MIMO Nonlinear Systems With Time-Varying Delays and Input Saturation," IEEE Transactions on Fuzzy Systems, vol. 24, no. 4, pp. 841-852, 2016.

[30] Y.-J. Liu and W. Wang, "Adaptive fuzzy control for a class of uncertain nonaffine nonlinear systems," Information Sciences, vol. 177, no. 18, pp. 3901-3917, 2007. 


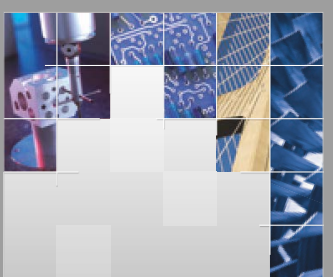

\section{Enfincering}
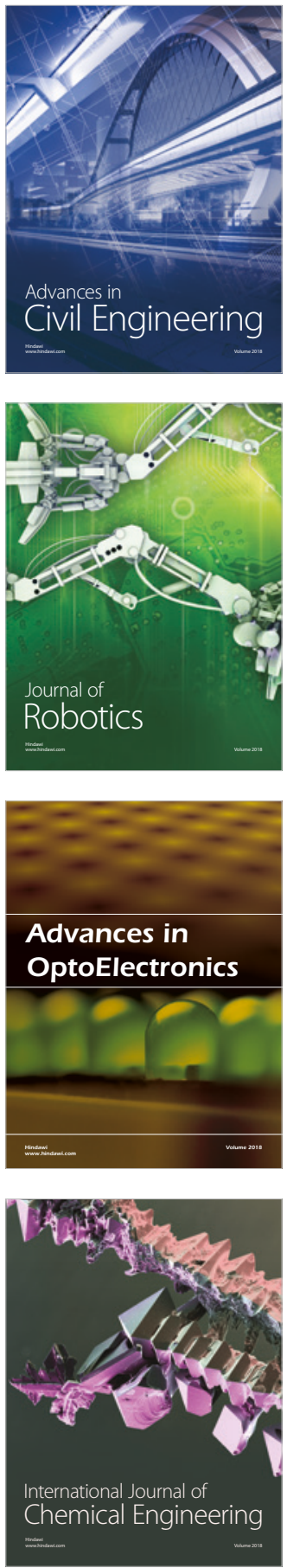

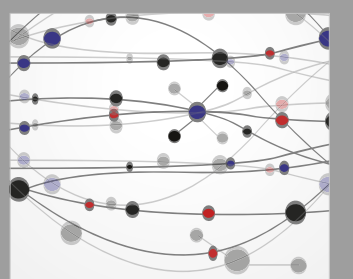

\section{Rotating \\ Machinery}

The Scientific World Journal

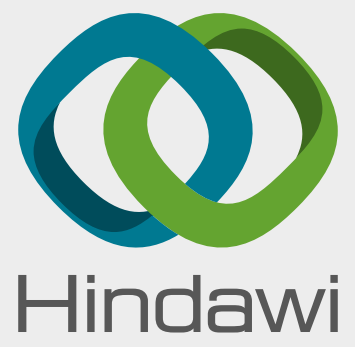

Submit your manuscripts at

www.hindawi.com
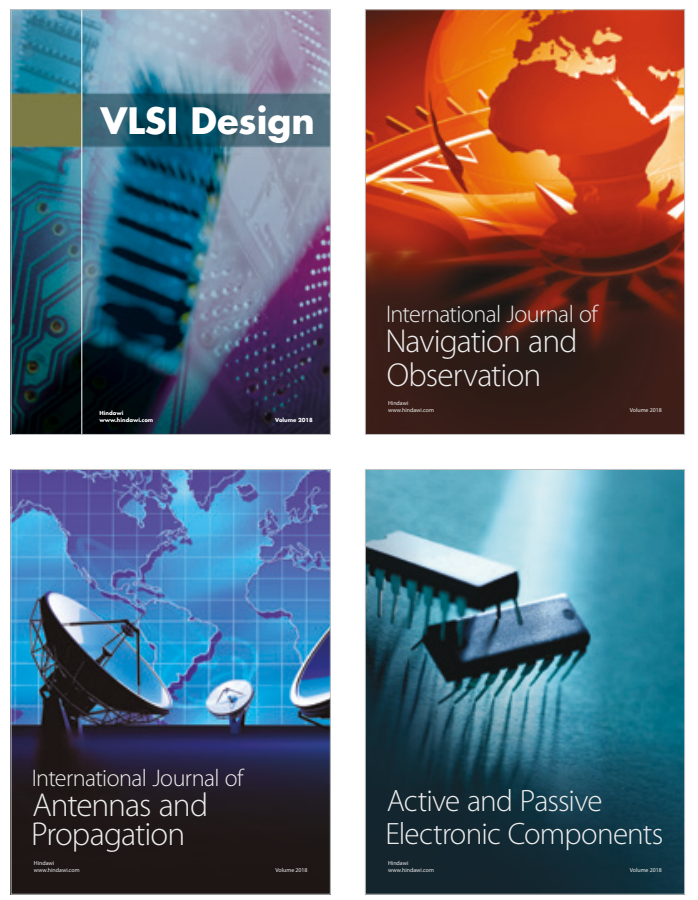
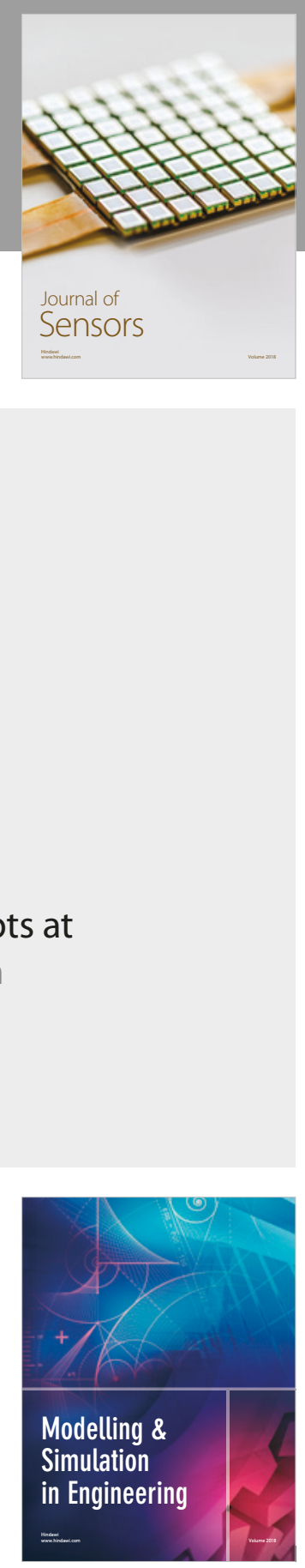

\section{Advances \\ Multimedia}
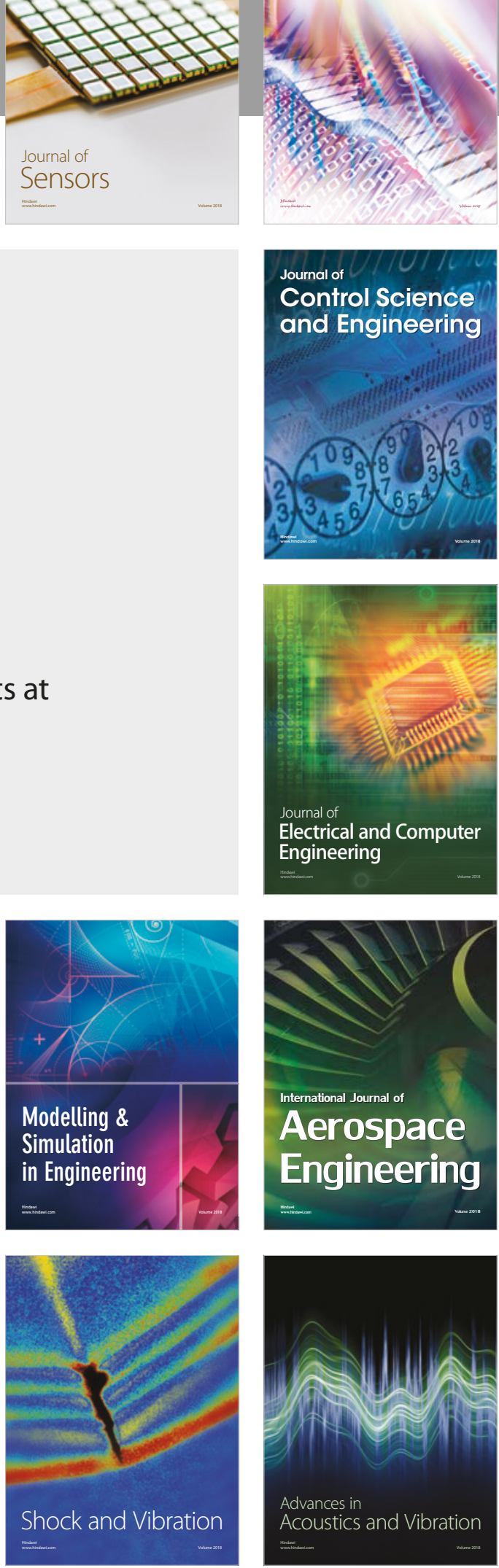